\title{
Finite element modelling of radial lentotomy cuts to improve the accommodation performance of the human lens
}

\author{
H.J. Burd*, G.S. Wilde \\ Department of Engineering Science, University of Oxford, Parks Road, Oxford OX1 3PJ, UK \\ *corresponding author; email: harvey.burd@eng.ox.ac.uk, tel. (01865) 273136 \\ Accepted for publication in Graefe's Archive for Clinical and Experimental Ophthalmology \\ $18^{\text {th }}$ November 2015
}

\begin{abstract}
Purpose The use of a femtosecond laser to form planes of cavitation bubbles within the ocular lens has been proposed as a potential treatment for presbyopia. The intended purpose of these planes of cavitation bubbles (referred to in this paper as 'cutting planes') is to increase the compliance of the lens, with a consequential increase in the amplitude of accommodation. The current paper describes a computational modelling study, based on three-dimensional finite element analysis, to investigate the relationship between the geometric arrangement of the cutting planes and the resulting improvement in lens accommodation performance. The study is limited to radial cutting planes.
\end{abstract}

Methods The effectiveness of a variety of cutting plane geometries is investigated by means of modelling studies conducted on a 45-year human lens.

Results The results obtained from the analyses depend on the particular modelling procedures that are employed. When the lens substance is modelled as an incompressible material, radial cutting planes are found to be ineffective. However, when a poroelastic model is employed for the lens substance, radial cuts are shown to cause an increase in the computed accommodation performance of the lens. In this case, radial cuts made in the peripheral regions of the lens have a relatively small influence on the accommodation performance of the lens; the lentotomy process is seen to be more effective when cuts are made near to the polar axis.

Conclusions When the lens substance is modelled as a poroelastic material, the computational results suggest that useful improvements in lens accommodation performance can be achieved, provided that the radial cuts are extended to the polar axis. Radial cuts are ineffective when the lens substance is modelled as an incompressible material. Significant challenges remain in developing a safe and effective surgical procedure based on this lentotomy technique.

Keywords: Lentotomy; Presbyopia; Accommodation; Modelling 


\section{Introduction}

The accommodation performance of the human eye declines with age, with the consequence that, from late middle-age onwards, the amplitude of accommodation becomes minimal. This limiting condition is known as presbyopia. There is interest in the possibility of developing new surgical procedures to correct for presbyopia. One particular proposed technique, termed lentotomy, consists of the use of a femtosecond laser to form intralenticular cuts. This procedure is based on the view that presbyopia is associated, principally, with an age-related increase in the stiffness of the lens.

The lentotomy process involves the use of focussed femtosecond laser pulses to generate cavitation bubbles (typically of the order of 5 microns in diameter) within the lens. The bubbles rapidly collapse after formation. The presence of these collapsed bubbles is thought to increase the local compliance of the lens substance with a consequential improvement in the amplitude of accommodation. In experimental lentotomy procedures, cavitation bubbles are usually arranged on internal planar or curved surfaces. These internal surfaces are typically referred to 'gliding planes' [10,13] or 'microincisions' [14]. The term 'cutting plane' is used in the current paper to describe these surfaces. If an appropriate geometric arrangement of cutting planes can be found, then it is anticipated that the deformations induced in the lens during the accommodation process will be increased and, as a consequence, the amplitude of accommodation will be enhanced.

Limited data are available in the research literature on the improvements in accommodation performance that are achievable using this technique. The data that do exist (e.g. $[9,12,13])$ typically relate to experiments on ex vivo lenses. Data from these experiments demonstrate that lentotomy cuts can lead to measurable changes in the compliance of the lens. However, the observed responses are typically small and with considerable variability between individual experiments.

The performance of the lentotomy process is clearly dependent on the geometric arrangement that is adopted for the cutting planes. Ripken et al. [12] describe a series of experiments on isolated porcine lenses in which various combinations of annular cuts (i.e. cutting planes aligned parallel to the lens equator), saggital cuts (in which cutting planes are aligned in radial directions) and cylindrical cuts (in which cavitation bubbles are introduced on a cylindrical surface centred on the polar axis of the lens) were applied to the lens. The compliance of the lens was assessed by measuring the induced changes in polar thickness when the isolated lens was spun (at rotational speeds of up to $1800 \mathrm{rpm}$ ) about its polar axis. These experiments typically indicate that treated lenses were more compliant than native lenses. Although annular and saggital cuts were shown to be effective, cylindrical cuts were found to have a detrimental effect on the observed compliance.

Schumacher et al. [13] describe an experimental study on isolated human lenses treated with lentotomy cuts arranged in a 'steering wheel pattern' (consisting of two cylindrical cuts, two annular cuts and a set of 12 saggital cuts). Two distinct mechanisms associated with the lentotomy process were observed. Firstly, immediately after the lens had been treated, there was an observed tendency for the thickness of the lens to increase and for the equatorial diameter to reduce. This behaviour is presumably associated with a redistribution of the residual stresses (i.e. internal stresses that exist in the absence of external forces) within the lens. Secondly, the compliance of the lens - determined using a spinning lens test - was 
typically found to be greater in the treated lenses, although the magnitude of the effect was relatively small.

These previous studies indicate the potential for modifying the compliance of the lens by the introduction of lentotomy cuts. There is considerable uncertainty, however, in the way in which any improvement in accommodation performance is influenced by the detailed geometry of the cuts. The search for effective arrangements of cutting planes by experimentation alone is impractical; a large number of experiments would be needed to explore all of the options. Moreover the natural variability between different samples of tissue is likely to mean that detailed interpretation of the experiments would be difficult.

The current paper describes a computational modelling procedure to complement experimental work in this area. The purpose of the current study is two-fold. Firstly, the modelling is intended to explore the improvements in accommodation performance that are potentially achievable using the lentotomy technique. Considerable uncertainty exists, for example, on whether the process is actually capable of delivering improvements in accommodation performance that are sufficiently large in magnitude to form the basis of a useful and reliable surgical technique. Secondly, the modelling is used to explore the influence that the size, number and spatial arrangements of the cuts might have on the overall accommodation performance of the lens. The current work is limited to the study of cuts in the lens along radial directions. This type of cut is referred to as 'saggital cut' in Ripken et al. [12] and Schumacher et al. [13]; in the current paper the terminology 'radial cut' is used.

Lentotomy seems most likely to yield positive results when conducted in relatively young presbyopes (on the basis that the lens nucleus continues to stiffen in later life $[7,16,17,18]$ ). The modelling studies described in the current paper are therefore based on a 45-year lens. At this age, the accommodation amplitude of the lens is substantially diminished but, at the same time, the stiffness of the lens nucleus is significantly lower that is the case in subsequent decades.

Previous mechanical models of the human lens (e.g. [3, 5, 6]) have typically assumed that the lens is axially symmetric about its polar axis. However, this symmetry is broken when the lens is cut along radial planes. This departure from axial symmetry means that a three dimensional modelling procedure is adopted in the current analyses.

\section{Finite element modelling procedures}

The modelling procedures adopted in the current study are based on the general framework described by Burd et al. [3] for axially symmetric finite element analyses of the accommodation performance of the natural lens. This previous model has been extended to three dimensions and further developed to incorporate recent data on the geometry and stiffness of the lens and also on the spatial variation in capsule thickness. The material models employed in the current analysis for the lens, capsule and zonules are based on a neoHookean constitutive model, formulated in the hyperelastic framework. This framework provides a rigorous means of incorporating the significant geometric changes that occur during the accommodation process. Numerical procedures have been developed to model the influence of radial lentotomy cuts on the accommodation performance of the lens. All calculations have been conducted using the finite element program ABAQUS v. 6.9-3 (Dassault Systèmes Simulia Corp., Providence, RI, USA), making use of the geometric nonlinear analysis facility. 


\section{Geometric model}

All 45-year lens analyses are conducted on lenses in which the finite element meshes were generated on a reference configuration based on the fully-accommodated (4D) outline developed by Hermans et al. [6] for a 45-year lens; see Fig 1. In this reference configuration the residual stresses (i.e. the stresses in the lens substance and capsule in the absence of external forces) are assumed to be zero. As a consequence of this assumption it is not possible to model any immediate shape changes induced by lentotomy cuts as has been, for example, observed in the tests reported by Schumacher et al. [13].

(a)

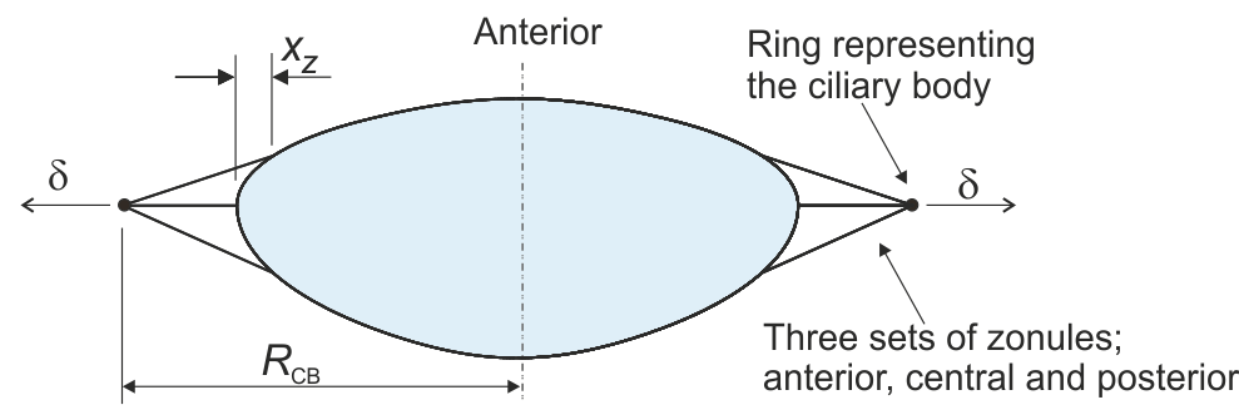

(b)

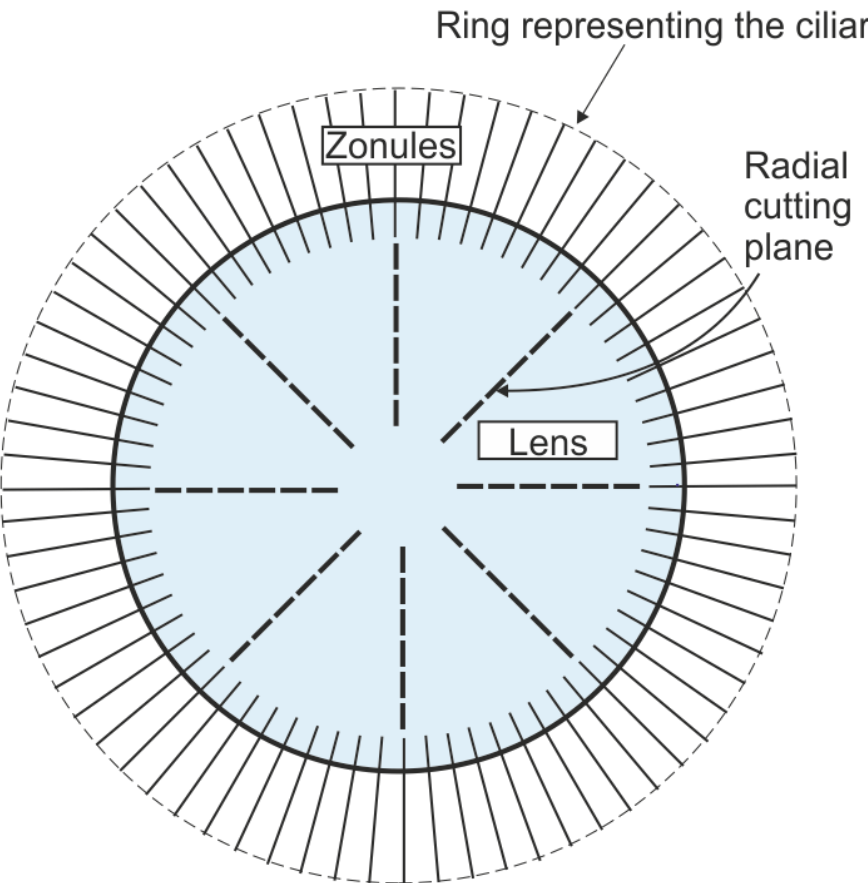

Fig. 1 Geometric model for the lens, zonules and ciliary body: (a) side view, (b) frontal view, showing the arrangement of radial cuts for the 8-cut scheme (i.e. 8 cutting planes are employed). The reference lens outline is based on the 45-year profile given in Hermans et al. [6]. The representation of the zonules is indicative only; in the finite element models, elements representing the anterior and posterior zonules are generated at an angular spacing of $1.5^{\circ}$ and elements representing the central zonules are generated at an angular spacing of $1.32^{\circ}$.

Spatial variations of stiffness typically exist in the human lens (e.g. $[7,16,18])$. In the young lens the nucleus is more flexible than the cortex but, in old age, this condition is reversed. Data from Wilde et al. [18] suggest that the nucleus and cortex have similar values of shear modulus at an age of about 42 years. On this basis, for the 45 -year lens that is the 
subject of the current study, the shear modulus of the nucleus and cortex are assumed to be equal. This approach, in which the lens is modelled as a homogeneous material, has the merit (from a practical perspective) that it is not necessary to include a distinct nucleus in the finite element model.

The lens capsule acts as a continuous structural membrane enclosing the lens substance. The thickness of the capsule is known to vary with radial position. In the current model, the capsule thickness is specified to vary spatially with position according to the Group A data (corresponding to samples with an average age of 36 years) in Barraquer et al. [2].

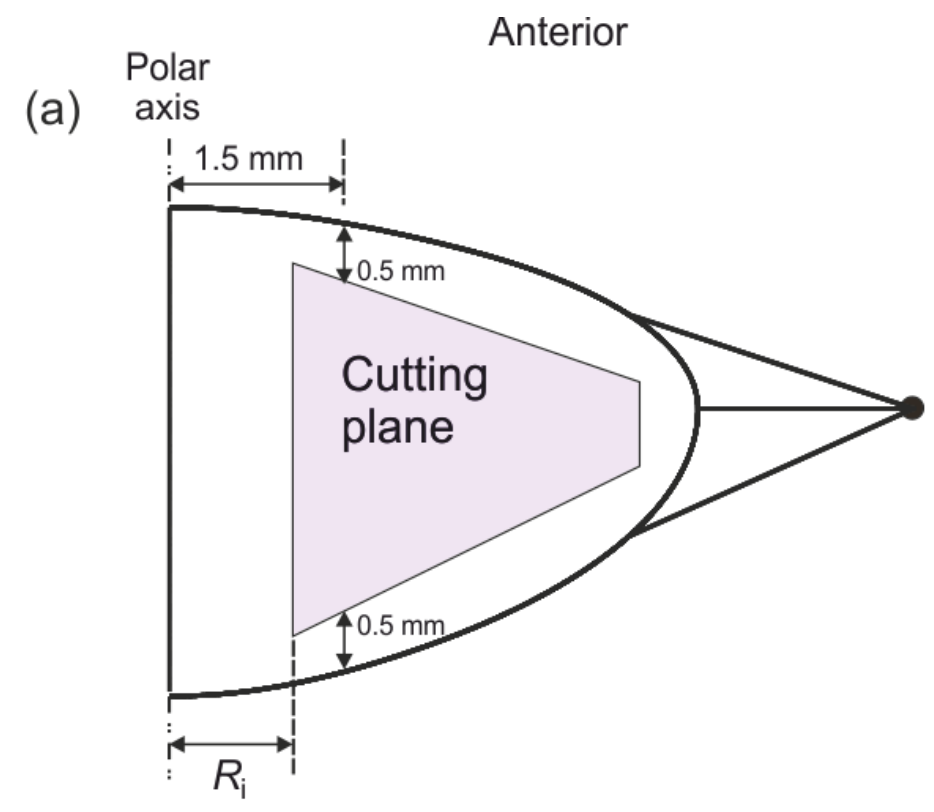

Anterior

(b)

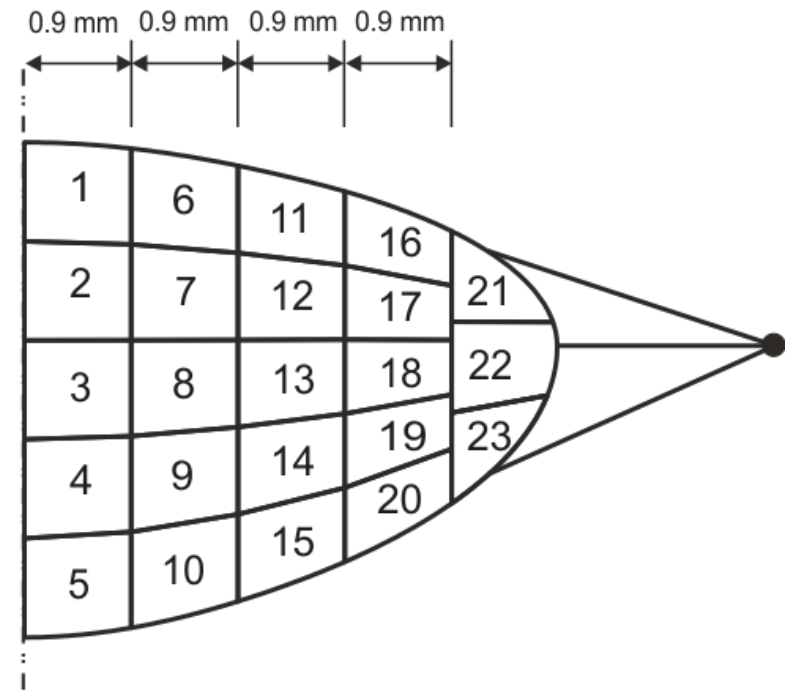

Fig. 2 (a) The standard cutting pattern geometry. The inner radius $R_{\mathrm{i}}$ is a variable that is adjusted in the parametric study; (b) notional cutting zones adopted for the exploratory pattern. The zone widths are spaced equally along each vertical line 
Following Burd et al. [3], three distinct sets of zonules, termed anterior, central and posterior, are represented in the model. The zonules connect the lens to a ring, radius $R_{C B}$, representing the connection of the zonules to the ciliary body. The radius of the ciliary body ring, $R_{C B}$, and the value of $x_{z}$ (that defines the location of the anterior and posterior zonule connections to the lens with respect to the lens equator) are determined from Equations (2) and (3) in Burd et al. [3] for a 45 -year lens (i.e. $R_{C B}=6.33 \mathrm{~mm}, x_{z}=0.589 \mathrm{~mm}$ ).

Two separate geometric arrangements of radial lentotomy cuts have been modelled. One set of studies has been conducted on a 'standard cutting pattern'. This consists of cutting planes with the geometry shown in Fig. 2a. The cuts are arranged symmetrically about the polar axis. Analyses have been conducted with different values of the 'inner radius', $R_{i}$. Two variants of this pattern have been studied; an 8-cut scheme (in which 8 individual cuts are arranged at an angular separation of $45^{\circ}$ around the polar axis as indicated in Fig 1b) and a 32-cut scheme (in which 32 individual cuts are arranged at an angular separation of $11.25^{\circ}$ around the polar axis).

A further study has been conducted using the 'exploratory pattern' shown in Fig 2b. In this study the cross-section of the lens is divided into 23 notional zones. Individual finite element calculations have been conducted to determine the performance of separate radial cuts in each of these zones. Calculations conducted on the exploratory pattern are all based on the use of a set of 32 radial cuts, equally spaced around the polar axis.

To minimise run times, advantage is taken of the symmetries that exist in the model. To exploit these symmetries, all finite element meshes were generated on a slice of the lens, as shown in Fig. 3. The angle $\theta$ indicated in Fig. 3 is referred to in this paper as the "wedge angle'.

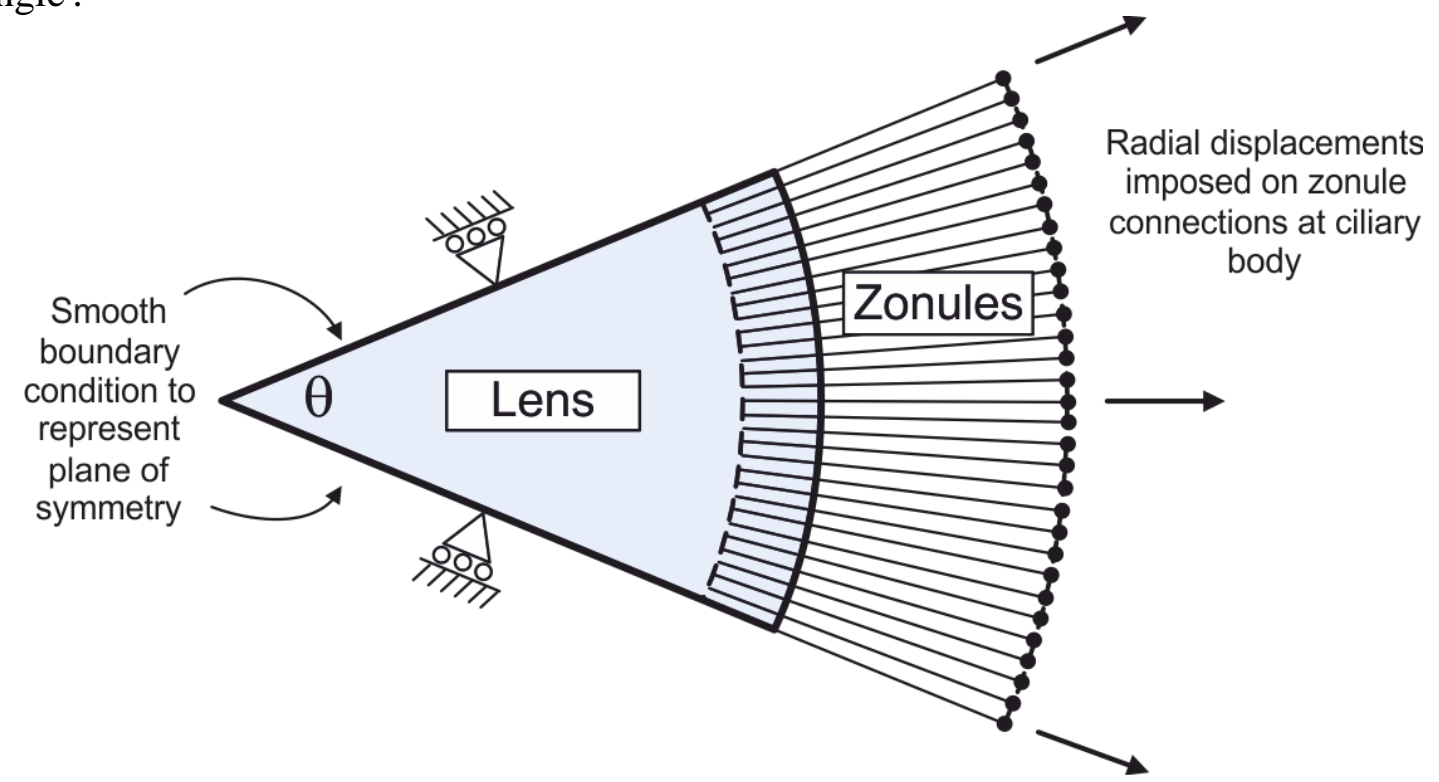

Fig. 3 Scheme adopted for the finite element models, viewed from the frontal direction. A wedge angle of $\theta=45^{\circ}$ was employed for the analysis of the standard cutting pattern and $\theta=11.25^{\circ}$ was employed for the analysis of the exploratory pattern.

The analyses are driven by prescribed changes in the radius of the ciliary body ring. These prescribed displacements cause the lens to stretch from the accommodated to the disaccommodated state. The prescribed ciliary body radial displacement, $\delta$, adopted in the 
analyses is taken from Equation (5) of Burd et al. [3] for a 45-year lens (i.e. $\delta=$ $0.2767 \mathrm{~mm})$.

\section{Material models for the lens, capsule and zonules}

The shear modulus adopted for the lens substance is based on the data (determined from spinning lens tests on decapsulated lenses) given in Wilde et al. [18]. The shear modulus adopted for the current analysis, based on the homogeneous model Model $\mathrm{H}$ in [18] for a 45year lens, is $1.38 \mathrm{kPa}$.

In previous numerical models of the accommodation performance of the lens, the lens substance is typically assumed to be incompressible. This incompressibility assumption is consistent with the data of Hermans et al. [4] that indicate that the volume of the nucleus is conserved during accommodation. However, the assumption of incompressibility has important implications in the current study, since local incompressibility can have a significant constraining effect on the lens substance in the neighbourhood of the lentotomy cuts. Some of the analyses in this paper are based on a modelling approach, referred to as 'Approach A', in which the lens is specified to be an incompressible neo-Hookean material. Incompressibility is represented, approximately, in the model by specifying a relatively high bulk modulus of $1.38 \mathrm{MPa}$ (i.e. a factor of 1000 greater than the shear modulus).

The use of an alternative approach, termed 'Approach B', in which a compressible neoHookean model is adopted for the lens, has also been explored. This model treats the lens as a poroelastic material (i.e. a two-phase mixture of an incompressible fluid and a compressible hyperelastic solid matrix). In this modelling procedure, the fluid is able to flow, freely, within the lens, but the lens capsule is specified to act as an impermeable barrier. This approach allows local volume changes to occur within the lens (e.g. to model the flow of fluid into the lentotomy cuts as they open up during the disaccommodation process). The outer surface of the lens is surrounded by a layer of 'hydrostatic' elements. These elements apply a hydrostatic pressure to the external surface of the lens to ensure that the total lens volume is conserved. (This external hydrostatic pressure is representative of the intralenticular pressure developed in the lens). A key feature of the poroelastic assumption is that the shear modulus of the compressible solid matrix is equal to the shear modulus of the equivalent, single phase, incompressible, model (i.e. a shear modulus of $1.38 \mathrm{kPa}$ ). A separate Poisson's ratio is required, however; for the current model this is taken to be $1 / 3$.

The capsule and the zonules are modelled (for Approaches A and B) as incompressible neo-Hookean materials. In each case the stiffness is specified by a one parameter, the shear modulus. For the capsule, the shear modulus was determined from the value of Young's modulus, $E_{c}$, given in Equation (4) of Burd et al. [3] (which is based on data given in Krag et al. [8]) for a 45-year lens, noting that for an incompressible material the shear modulus is $E_{c} / 3$ (i.e. the capsule shear modulus is set to $0.483 \mathrm{MPa}$ in the current analysis).

The three sets of of zonules (anterior: posterior: central) have values of stiffness assigned to them in the ratio 6:3:1 (Burd et al. [3]). Appropriate stiffness values were determined using a procedure, described in [3], in which the stiffness of the zonules in a 29-year axially symmetric finite element model is adjusted so that the computed lens equatorial displacement induced during the disaccommodation process is equal to corresponding displacement determined from the MRI data in Strenk et al. [15]. In this separate calibration analysis, the reference configuration was based on the fully-accommodated 29-year lens outline given in 
Hermans et al. [6]. Lens stiffness data were determined from the Model D data given in Wilde et al. [18]. The values of zonule stiffness determined in this way are listed in Table 1. These values of zonule stiffness are adopted in all of the 45-year lens calculations described in the current paper.

It is anticipated that the lens capsule and zonules, being thin structures, are unable to sustain compressive stresses of any significant magnitude. The constitutive models were therefore specified to ensure that compressive stresses cannot occur in these elements.

\begin{tabular}{|l|l|l|l|}
\hline Zonule group & Anterior & Central & Posterior \\
\hline Stiffness $(\mathbf{m N})$ & 303.8 & 50.6 & 151.9 \\
\hline
\end{tabular}

Table 1. Stiffness data for the zonules adopted for all of the 45-year analyses, given as the product of the total cross-sectional area of the zonules (for the entire circumference) and the shear modulus.

\section{Finite element meshes}

Finite element meshes were generated using 10-noded tetrahedral elements for the lens substance, 6-noded triangular membrane elements for the capsule and 2-noded bar elements to represent the zonules. The capsule mesh was created so that element edges followed ring segments corresponding to the anterior, equatorial, and posterior zonule attachment latitudes. Each node lying on these ring segments served as an attachment for an individual bar element, so that the three-dimensional model would match the axially symmetric case as closely as possible in this respect. The desired local capsule thickness was specified at each of the nodes on the lens outer surface.

Initial analyses conducted to investigate the accommodation performance of the untreated lens were based on the mesh shown in Fig. 4. Similar forms of mesh were adopted for the analyses in which lentotomy cuts are included in the model.

(a)

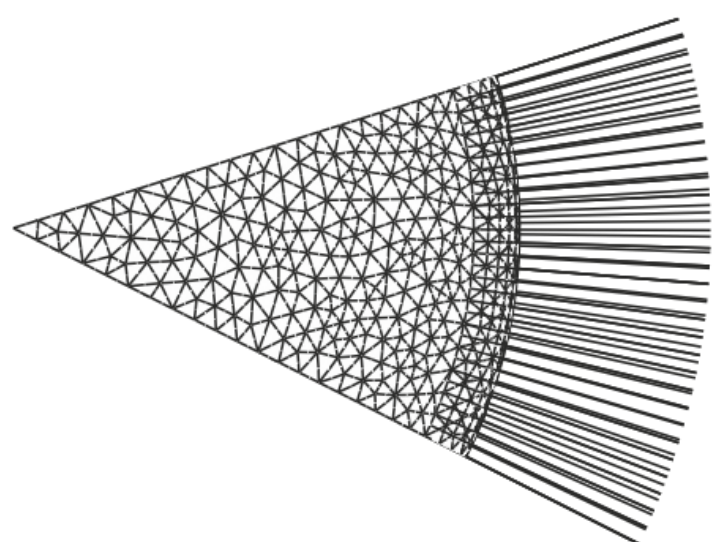

(b)

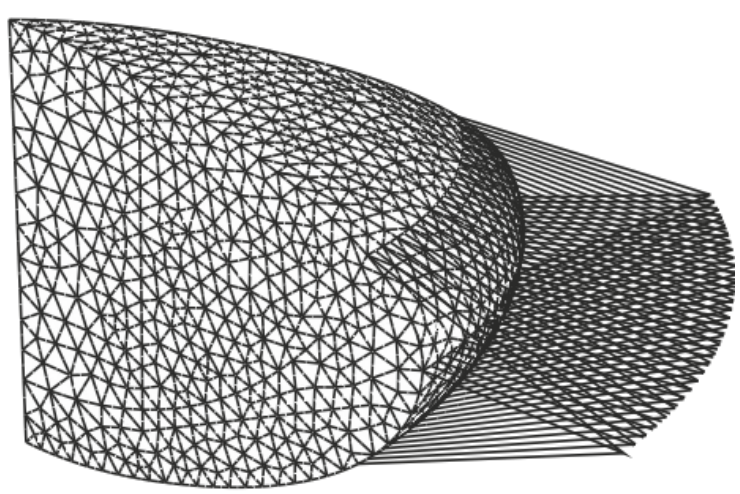

Fig. 4. Finite element mesh for the untreated lens: (a) view of anterior surface, (b) isometric view. Wedge angle, $\theta=45^{\circ}$. A total of 26005 continuum elements to model the lens substance, 1304 membrane elements to model the capsule and 97 bar elements to model the zonules are employed in the mesh. 


\section{Optical analysis}

Lens optical power $(L O P)$ is determined from the numerical results using the conventional thick lens formula,

$$
L O P=\frac{n_{l}-n_{a}}{r_{a}}+\frac{n_{l}-n_{a}}{r_{p}}-t \frac{\left(n_{l}-n_{a}\right)^{2}}{n_{l} r_{a} r_{p}}
$$

where the refractive index of the lens, $n_{l}$, is 1.42 and the refractive index of the aqueous and vitreous, $n_{a}$, is 1.336 . The radius of curvature of the anterior and posterior surfaces are $r_{a}$ and $r_{p}$ respectively (where both radii are treated as positive) and the polar thickness is $t$. Values of $r_{a}$ and $r_{p}$ are determined from the finite element results by finding the radius of the sphere that provides a best fit with the surface nodes for a circular aperture of radius $1.5 \mathrm{~mm}$. The lens accommodation amplitude, $\triangle L O P$, is the change in $L O P$ between the initial (fully accommodated ) and stretched (disaccommodated) states.

The optical results presented later in this paper relate to values of $\triangle L O P$ computed using Equation (1). It should be noted that the objective accommodation amplitude of the whole eye (e.g. as calculated using a schematic eye) is typically lower than the lens accommodation amplitude determined in this way. Data in Rabbett [11] for example, suggest that the objective accommodation amplitude can be estimated as $0.81 \times \triangle L O P$. A factor should therefore be applied to the computed values of $\triangle L O P$ presented later in this paper to estimate the expected objective accommodation amplitude. Following [11] a factor of 0.81 is suggested.

\section{Computed zonular force}

The computed finite element results may be used to determine the force acting on each bar element (corresponding to a notional group of zonules) at the point where it connects with the ciliary body ring. The radial components of these forces may be summed to give the radial zonular force required to stretch the lens slice from the accommodated to the dissaccommodated state. The total radial zonular force $\Delta F_{Z}$ (corresponding to the entire circumference of the lens) is determined by applying a factor of $360 / \theta$ (where $\theta$ is the wedge angle in degrees) to the radial zonular force determined by summing the radial forces determined from the finite element analysis.

\section{Numerical studies on the influence of radial lentotomy cuts}

The development of realistic modelling procedures to represent the influence of the lentotomy cuts on the accommodation performance of the lens presents a considerable challenge. The authors (in preliminary work) have tried a variety of procedures, with variable results. In the current paper, two separate modelling approaches are adopted. For analyses conducted using Approach A (incompressible lens) the cutting planes are represented by thin zones of material (termed 'cutting zones') with a reduced shear and bulk modulus. For Approach B (poroelastic lens) an alternative approach is adopted in which the cutting planes are modelled, explicitly, by cut planes generated in the finite element mesh. In this case, it is assumed that the internal volumes that are generated as the cuts open up (during the disaccommodation process) become filled with fluid from the surrounding tissue. Since this process involves local volume changes, this type of behaviour cannot be modelled with an 
incompressible lens substance (Approach A). Three separate modelling studies have been conducted as specified below.

Standard cutting pattern analysed using Approach A (incompressible lens)

The performance of the 'standard cutting pattern' with $R_{\mathrm{i}}=1.5 \mathrm{~mm}$ (Fig 2a) has been investigated using Approach A. The analyses are specified in Table 2.

\begin{tabular}{|l|l|l|c|c|c|}
\hline Model & $\begin{array}{l}\text { Total } \\
\text { number of } \\
\text { cuts }\end{array}$ & Value of $\boldsymbol{t}_{\text {cut }}$ & $\boldsymbol{\Delta L O P}$ & $\begin{array}{c}\text { Change in } \\
\boldsymbol{\Delta} \boldsymbol{L O P} \text { due to } \\
\text { cutting }\end{array}$ & $\boldsymbol{\Delta F}_{\boldsymbol{Z}}$ \\
\hline A0 & \multicolumn{2}{|l|}{ Untreated lens } & $2.79 \mathrm{D}$ & & $49.4 \mathrm{mN}$ \\
\hline \hline A1 & 8 & 90 microns & $2.64 \mathrm{D}$ & $-0.15 \mathrm{D}$ & $49.1 \mathrm{mN}$ \\
\hline A2 & 8 & 360 microns & $2.08 \mathrm{D}$ & $-0.71 \mathrm{D}$ & $48.4 \mathrm{mN}$ \\
\hline A3 & 32 & 90 microns & $2.13 \mathrm{D}$ & $-0.66 \mathrm{D}$ & $48.5 \mathrm{mN}$ \\
\hline
\end{tabular}

Table 2. Analyses conducted on the standard cutting pattern with $R_{i}=1.5 \mathrm{~mm}$ using Approach A (incompressible lens). The parameter $t_{c u t}$ is the thickness of the zone of reduced-stiffness material used to model the treated zones. The parameter $\triangle L O P$ is the computed lens accommodation amplitude. Data on $\triangle L O P$ are given in units of dioptres. $\Delta F_{Z}$ is the value of total radial zonular force required to stretch the lens from the accommodated to the disaccommodated state.

Two geometric arrangements (8-cut scheme and 32-cut scheme) have been investigated. The analyses on the 8-cut scheme (i.e. cuts at a circumferential separation of $45^{\circ}$ ) are based on the lens slice geometry shown in Fig. 5a and the analysis on the 32-cut scheme (i.e. cuts at a circumferential separation of $11.25^{\circ}$ ) adopted the geometry depicted in Fig. 5b. A reference analysis, Model A0, was conducted (using the mesh in Fig. 4) to compute the performance of the untreated lens.

(a)

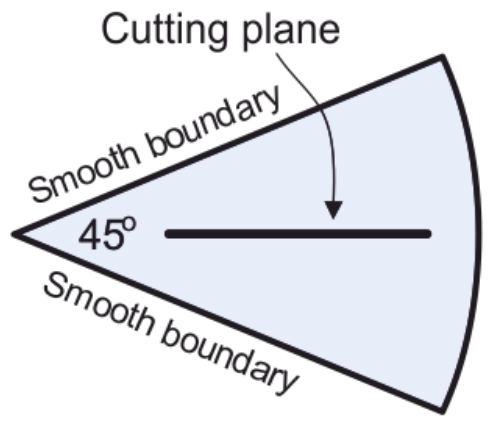

(b)

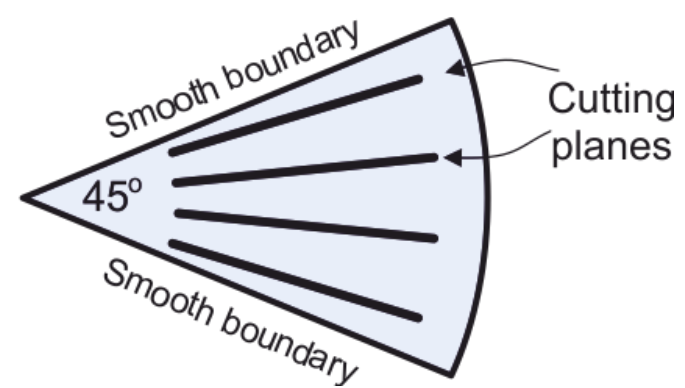

Fig. 5 Schematic diagram of the modelling scheme adopted for the standard cutting pattern, analysed using Approach A (incompressible lens). View from the frontal direction. Zonules and ciliary body ring, omitted from these diagrams for clarity, are as indicated in Fig. 3.

Radial lentotomy cuts are included in the model by specifying cutting zones, thickness $t_{\text {cut }}$, within the finite element mesh. The lens material within the cutting zones is assigned values of shear and bulk modulus that are $10 \%$ of the shear and bulk modulus, respectively, of the natural lens substance. It should be noted that the values of $t_{c u t}$ adopted in these models (see Table 2) are significantly larger than typical dimensions of the cavitation bubbles induced during the lentotomy process. The reasons for adopting these relatively large values 
of $t_{c u t}$ are as follows. Firstly, it is anticipated that very thin cutting zones will have a negligible effect in this type of model (as a consequence of the incompressible behaviour of the surrounding lens substance). Secondly, it is expected, that the lentotomy process will influence the material surrounding the cutting zone, as well as within the cutting zone itself. Furthermore, very thin zones would require refined meshes to be used in the neighbourhood of the cut; this would lead to a large number of elements and lengthier run-times.

\section{Standard cutting pattern analysed using Approach B (poroelastic lens)}

In this set of analyses, the lens is represented by a poroelastic model and cutting planes are included in the model by specifying surfaces within the finite element mesh that are adjacent but disconnected. In the accommodated state, the two surfaces are coincident. As the lens is stretched, however, the surfaces are able to move apart. This is regarded as a more realistic procedure than the cutting zone approach adopted in the Approach A calculations.

Analyses have been conducted for the standard cutting pattern (Fig. 2) for both the 8-cut and 32-cut schemes. These analyses are based on the modelling scheme shown in Fig. 6 and are specified in Table 3. This analysis scheme involves cutting planes on the boundary of the lens slice being modelled. This contrasts with the modelling scheme adopted in the Approach A calculations (Fig. 5) in which all cutting zones are internal. The arrangement shown in Fig. 6 has been adopted for the Approach B calculations, for convenience, on the basis that is relatively straightforward to generate cut planes that coincide with an external boundary.

(a)

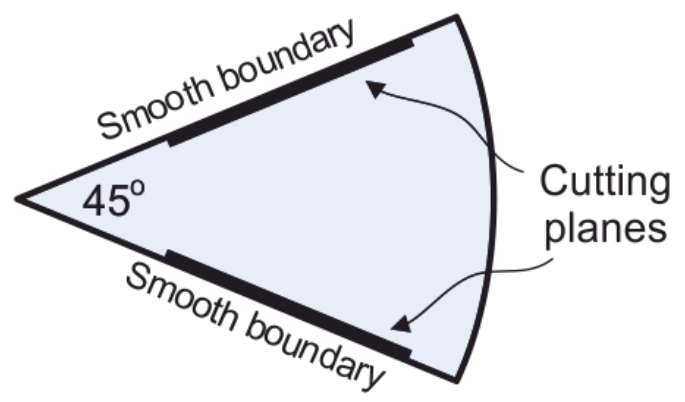

(b)

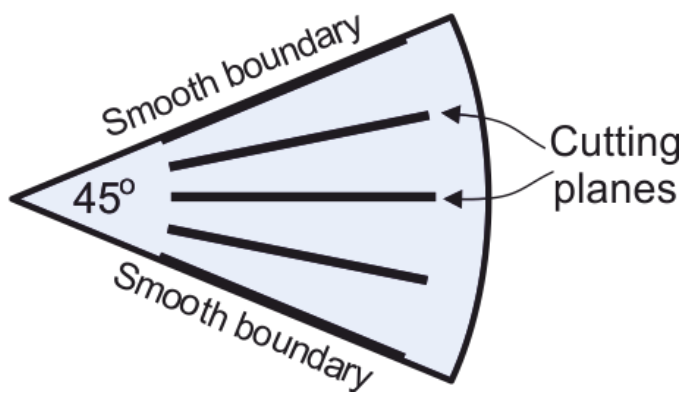

Fig. 6. Schematic diagram of the modelling scheme adopted for the standard cutting pattern, analysed using Approach B (poroelastic lens): (a) 8-cut scheme, (b) 32-cut scheme. View from the frontal direction. Zonules and ciliary body ring, omitted from these diagrams for clarity, are as indicated in Fig. 3.

\begin{tabular}{|l|l|l|l|l|c|}
\hline Model & $\begin{array}{l}\text { Total number } \\
\text { of cuts }\end{array}$ & Value of $\boldsymbol{R}_{\boldsymbol{i}}$ & $\boldsymbol{\Delta L O P}$ & $\begin{array}{l}\text { Change in } \boldsymbol{\Delta} \boldsymbol{L O P} \\
\text { due to cutting }\end{array}$ & $\boldsymbol{\Delta F}_{\boldsymbol{Z}}$ \\
\hline B0 & Untreated lens & $2.97 \mathrm{D}$ & & $49.2 \mathrm{mN}$ \\
\hline \hline B1 & 8 & $1.5 \mathrm{~mm}$ & $3.28 \mathrm{D}$ & $0.31 \mathrm{D}$ & $48.9 \mathrm{mN}$ \\
\hline B2 & 8 & $1.0 \mathrm{~mm}$ & $3.62 \mathrm{D}$ & $0.65 \mathrm{D}$ & $48.8 \mathrm{mN}$ \\
\hline B3 & 8 & $0.5 \mathrm{~mm}$ & $4.03 \mathrm{D}$ & $1.06 \mathrm{D}$ & $48.7 \mathrm{mN}$ \\
\hline B4 & 8 & $0.0 \mathrm{~mm}$ & $4.77 \mathrm{D}$ & $1.8 \mathrm{D}$ & $48.5 \mathrm{mN}$ \\
\hline B5 & 32 & $1.5 \mathrm{~mm}$ & $3.38 \mathrm{D}$ & $0.41 \mathrm{D}$ & $48.8 \mathrm{mN}$ \\
\hline
\end{tabular}

Table 3. Analyses conducted for the standard cutting pattern using Approach B (poroelastic lens). Data on $\triangle L O P$ are given in units of dioptres. $\Delta F_{Z}$ is the value of total radial zonular force required to stretch the lens from the accommodated to the disaccommodated state. 


\section{Exploratory pattern analysed using Approach B (poroelastic lens)}

A third set of analyses has been conducted on the exploratory pattern (Fig. 2b) to investigate the regions within the lens that are most likely to lead to positive results when treated with radial lentotomy cuts. These analyses have been conducted for the 32-cut scheme only. They are all based on the use of Approach B (poroelastic lens) with cut planes generated explicitly within the finite element mesh. The meshes were generated on the geometric model shown in Fig. 7.

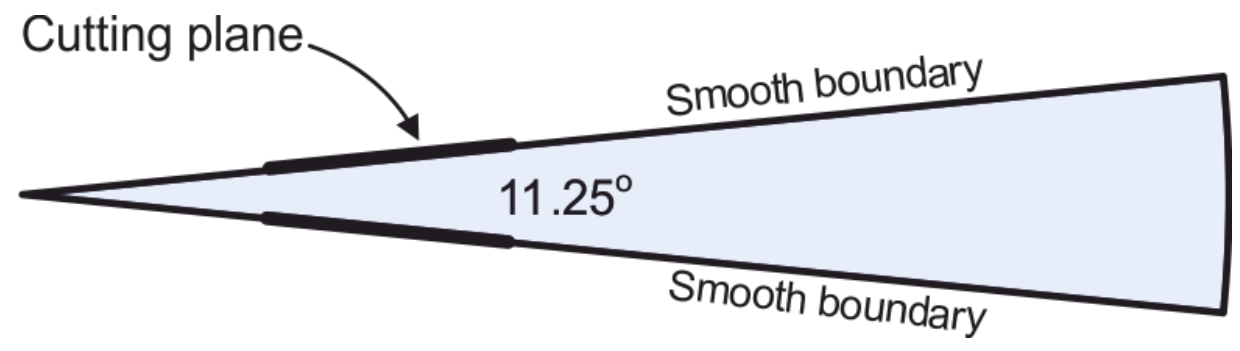

Fig. 7 Schematic diagram of the modelling procedure adopted for the exploratory pattern, 32-cut scheme, analysed using Approach B (poroelastic lens). View from the frontal direction. Zonules and ciliary body ring, omitted from the diagram for clarity, are as indicated in Fig. 3. Cutting planes were modelled on the two smooth boundaries. The cutting planes shown in the figure corresponds to any one of the zones 6-10, in Fig $2 \mathrm{~b}$.

\section{Results}

Standard cutting pattern analysed using Approach A (incompressible lens)

Results obtained from the Approach A analyses on the standard cutting pattern are given in Table 2. The untreated lens (Model A0) was found to have a value of $\triangle L O P$ of 2.79D. On the basis of the 0.81 factor given in Rabbett [11], this corresponds to an objective accommodation amplitude of 2.26D. This is consistent with published data on the objective accommodation performance of a 45-year subject (e.g. [1]).

Table 2 indicates that the computed accommodation amplitudes of the lenses that had been treated with lentotomy cuts are, in all cases, less than the amplitude of the untreated lens. Moreover, increasing the number of cutting planes from 8 to 32 (compare A1 and A3) and increasing the value of $t_{c u t}$ (compare A1 and A2) have the effect of reducing the accommodation performance of the lens. These counter-intuitive results are strongly conditioned by the incompressible behaviour of the lens material within and near the cutting zones. The total volume of the cutting zone cannot change significantly as the lens is stretched. Any radial or circumferential (tensile) strains induced in the cutting zone will be therefore be accompanied by compressive strains in the axial direction. Any additional axial strains will tend to counteract the reduction in lens surface curvature that develops as the lens is stretched into its disaccommodated form. As a consequence, the model appears to demonstrate that lentotomy cuts have a detrimental effect on the accommodation performance of the lens.

Table 2 also indicates values for the computed total radial zonular force $\Delta F_{Z}$ required to stretch the lens into its disaccommodated form. For the untreated lens, the computed value of $\Delta F_{Z}$ is $49.4 \mathrm{mN}$. This value of radial force is comparable to results obtained from previous computational models of the accommodation performance of the human lens (e.g. [5]). It is 
clear from the data in Table 2 that the total radial force required to stretch the lens is not significantly influenced by the presence of the lentotomy cuts.

The incompressible assumption, as adopted in Approach A, is relatively straightforward to implement. However, it is thought this it is unlikely to provide a realistic representation of the mechanical behaviour of the lens substance within and near to the cuts. It seems plausible, for example, that local volume changes will occur, during the accommodation process, as a result of local fluid flows. Local volume changes are likely to have a significant influence on the way in which the lentotomy cuts influence the accommodation performance of the lens.

\section{Standard cutting pattern analysed using Approach B (poroelastic lens)}

Results obtained using Approach B (poroelastic model) to investigate the performance of the standard cutting pattern are given in Table 3. The computed accommodation amplitude for the untreated lens (Model B0) is similar to the corresponding value computed using Approach A (incompressible lens) (see Model A0, Table 2). Furthermore, the computed values of $\Delta F_{Z}$ for Models $\mathrm{B} 0$ and $\mathrm{A} 0$ are similar. These comparisons suggests that the accommodation performance of the untreated lens is insensitive to the particular approach that is used to model the material behaviour of the lens substance. Similar to the Approach A analyses, the lentotomy cuts for Approach B are seen to have little influence on the computed values of $\Delta F_{Z}$.

The data in Table 3 indicate that lentotomy cuts invariably cause the computed lens accommodation amplitude to be increased. The improvements in accommodation performance develop steadily as the inner radius is reduced. For $R_{i}=0 \mathrm{~mm}$ (Model B4) a useful increase in lens accommodation amplitude of 1.8D is achieved (for the 8-cut scheme). However, this is at the expense of cutting the lens within the central zone; cutting in this area is undesirable because of the risk of interfering with the optical performance of the lens. Models B1 and B5 indicate that, for $R_{\mathrm{i}}=1.5 \mathrm{~mm}$, increasing the number of radial cuts from 8 to 32 leads to a small improvement in accommodation amplitude.

\section{Exploratory pattern analysed using Approach B (poroelastic lens)}

Results on $\triangle L O P$ obtained from the 23 separate analyses on the exploratory pattern are tabulated in Table 4 and plotted in Fig 8. These data indicate that cuts in the peripheral regions (i.e. zones 11-23) have a positive, but small, influence on the computed accommodation amplitude. Lentotomy cuts in these areas seem unlikely to lead to significant improvements in accommodation performance; this observation is consistent with the computed performance of the standard cutting pattern, for $R_{\mathrm{i}}=1.5 \mathrm{~mm}$ (Table 3 ). 


\begin{tabular}{|l|l||l|l||l|l||l||l|}
\hline Zone & $\Delta(L O P)$ & Zone & $\Delta(L O P)$ & Zone & $\Delta(L O P)$ & Zone & $\Delta(L O P)$ \\
\hline 1 & -0.0555 & 7 & 0.0957 & 13 & 0.0519 & 19 & 0.0177 \\
\hline 2 & 0.0871 & 8 & 0.0932 & 14 & 0.0609 & 20 & 0.0156 \\
\hline 3 & 0.0937 & 9 & 0.1565 & 15 & 0.0752 & 21 & 0.0041 \\
\hline 4 & 0.1526 & 10 & 0.4068 & 16 & 0.0118 & 22 & 0.0057 \\
\hline 5 & -0.1527 & 11 & 0.0367 & 17 & 0.0159 & 23 & 0.0043 \\
\hline 6 & 0.1401 & 12 & 0.0474 & 18 & 0.0168 & & \\
\hline
\end{tabular}

Table 4; Computed data on $\triangle L O P$ for each individual notional cutting zone in the exploratory pattern. Analyses conducted using Approach B (poroelastic lens). Data are given in units of dioptres and are plotted in Fig. 8. See Fig. $2 b$ for the zone numbering scheme.
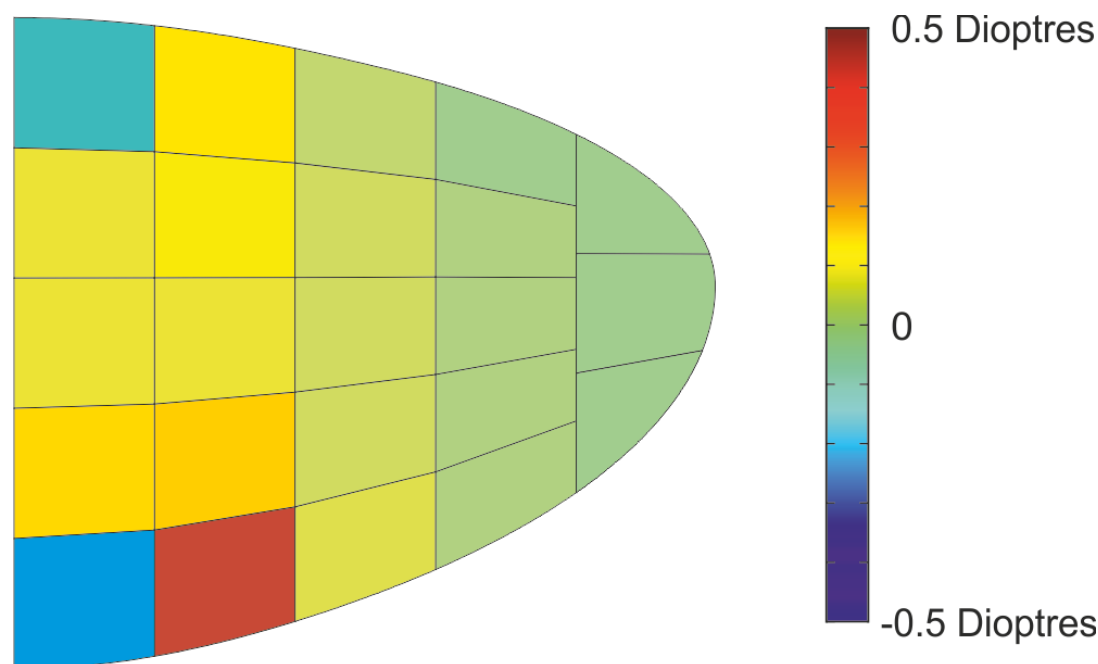

Fig. 8. Values of $\triangle L O P$, from analyses on the exploratory pattern, Approach B (poroelastic lens). The colours indicate values of $\triangle L O P$ in units of dioptres.

The influence of lentotomy cuts near to the central regions of the lens is more marked. Cutting in zone 10, for example, delivers a relatively large, positive, change in $\triangle L O P$. However, cutting in the two surface zones adjacent to the polar axis (zones 1 and 5) causes $\triangle L O P$ to reduce. It appears that, in the central regions of the lens, the computed values of $\triangle L O P$ are highly sensitive to minor variations in the spatial location of the cuts.

\section{Discussion}

The authors do not consider that there is sufficient evidence to determine whether the incompressible lens model (Approach A) or the poroelastic lens model (Approach B) provide a realistic model of the mechanics of the lentotomy process. The modelling results show, however, that when an incompressible model for the lens is employed, radial lentotomy cuts have a detrimental effect on the computed accommodation performance. In contrast, when the poroelastic approach is used, the computed accommodation performance of the lens is shown to be enhanced by the cuts. It seems plausible (to the authors) that imposing local incompressibility at the cuts (Approach A) over-constrains the model, while having no 
interaction between the poroelastic and fluid phases (Approach B) may result in a model that is unrealistically under-constrained.

The analysis of the standard cutting pattern using Approach B suggests that the lentotomy process is capable of increasing the accommodation amplitude of a 45-year lens. However, for the case where $R_{\mathrm{i}}=1.5 \mathrm{~mm}$, the improvement in $\triangle L O P$ is relatively small $(0.41 \mathrm{D}$ for 32 radial cuts). Cutting geometries of this sort, that are confined to the peripheral regions, are desirable on the basis that cutting in the optical zone is avoided. However, the computed performance of cuts made in the peripheral regions of the lens is poor. The performance of the lentotomy process is seen to be more effective when cuts are made near to the polar axis. For example, for the standard cutting pattern modelled with Approach $\mathrm{B}$, for $R_{\mathrm{i}}=0.0 \mathrm{~mm}$, the improvement in $\triangle L O P$ is $1.8 \mathrm{D}$ (with an 8-cut scheme). It seems plausible (although this has not been tried) that a 32-cut scheme for this case will provide a further enhancement.

Detailed studies on the individual effectiveness of a set of 23 notional cutting zones, (the exploratory pattern) conducted using Approach B, indicate that zones near the polar axis typically have a more significant effect on performance than those that are located in the peripheral regions. This finding is broadly consistent with the performance of the standard cutting pattern computed using Approach $\mathrm{B}$. The results of the exploratory pattern modelling study do not, however, identify any regions of the lens that are likely to respond particularly strongly to lentotomy cuts.

The general impression gained from these results is that radial lentotomy cuts act to increase the axial compression developed in the lens during the disaccommodation process. It is noted that the optics of the lens are sensitive to exactly where any increased compression is achieved. If a significant enhancement in compression occurs on the optical axis, then the accommodation performance of the lens is likely to be improved. Excess compression in the peripheral regions of the lens relative to the centre, however, will be counter-productive.

In broad terms, disaccommodation of a young lens is associated with compression in the lens substance near the polar axis and a radial movement of the lens substance in the peripheral regions. The radial cutting process investigated in this paper appears to enhance these movements in the following ways:

(a) The presence of the radial cuts reduces the circumferential constraints on the lens substance. This causes a reduction in resistance to radial movements of the lens substance when the lens is stretched during disaccommodation.

(b) The reduction of circumferential stresses during disaccommodation causes, induced by the radial cuts, causes the radial stresses acting on the inner portions of the lens to increase. These increased radial stresses, in turn, cause increased compression in the lens substance near the axis.

The modelling described in this paper is regarded as being preliminary on the basis that most of the results are based on a poroelastic model that lacks experimental validation. However, the results from this modelling study suggest that the development of radial cutting protocols that are capable of forming a useful surgical technique presents a formidable, and perhaps an insuperable, challenge. It is desirable (from the perspective of safety and optical quality) for the central region of the lens to be left free of cuts. However, the modelling results show that the benefits of cutting in the peripheral regions are small. Useful 
improvements in lens accommodation amplitude can be achieved, however, if the radial cuts are extended to the polar axis.

The natural stiffening of the lens provides a further challenge to the lentotomy procedure. The data of Wilde et al. [18] for example, indicate that the stiffness of the lens nucleus increases continuously during life. This suggests that any improvements in the accommodation performance that are achieved by radial cutting in a relatively young presbyope are likely to be lost in subsequent years.

The broad conclusions of this study present a rather pessimistic assessment of the likely effectiveness of lentotomy procedures based on radial cuts alone. It is acknowledged that shortcomings may exist in the modelling procedures being used. It is also plausible that the lens may be more sensitive to other geometric forms of cutting geometry than the ones considered in this study. Furthermore, radial cuts may prove to be effective when combined with other cutting geometries.

Funding: The research described in this paper was supported by a research grant from Rowiak $\mathrm{GmbH}$. The sponsor had no role in the design or conduct of the research.

Conflict of Interest: The authors declare that they have no conflict of interest.

Ethical approval: This article does not contain any studies with human participants or animals performed by any of the authors.

\section{References}

1. Anderson HA, Hentz G, Glasser A, Stuebing KK, Manny, RE (2008). Minus-lensstimulated accommodative amplitude decreases sigmoidally with age: a study of objectively measured accommodative amplitudes from age 3. Invest Ophth Vis Sci 49 (7): 2919-2926

2. Barraquer RI, Michael R, Abreu R, Lamarca J, Tresserra F (2006). Human lens capsule thickness as a function of age and location along the sagittal lens perimeter. Invest Ophth Vis Sci 47 (5): 2053-2060

3. Burd HJ, Judge SJ, Cross JA (2002). Numerical modelling of the accommodating lens. Vision Res 42 (18): 2235-2251

4. Hermans E, Dubbelman M, van der Heijde GL, Heethaar R (2007). The shape of the human lens nucleus with accommodation. J Vision 7 (10)

5. Hermans EA, Dubbelman M, van der Heijde GL, Heethaar RM (2006). Estimating the external force acting on the human eye lens during accommodation by finite element modelling. Vision Res 46 (21): 3642-3650

6. Hermans EA, Dubbelman M, van der Heijde GL, Heethaar RM (2008). Change in the accommodative force on the lens of the human eye with age. Vision Res, 48 (1): 119-126

7. Heys KR, Cram SL, Truscott RJW (2004) Massive increase in the stiffness of the human lens nucleus with age: the basis for presbyopia? Mol Vis 10: 956-963

8. Krag S, Andreassen TT, Olsen T (1996) Elastic properties of the lens capsule in relation to accommodation and presbyopia. Invest Ophth Vis Sci 47 (5): Abstract no. 774.

9. Krueger RR, Sun XK, Stroh J, Myers R. (2001) Experimental increase in accommodative potential after neodymium:yttrium-aluminum-garnet laser photodisruption of paired cadaver lenses. Ophthalmology 108 (11): 2122-2129 
10. Lubatschowski H, Schumacher S, Fromm M, Wegener A, Hoffman H, Oberheide U, Gerten G (2010) Femtosecond lentotomy: generating gliding planes inside the crystalline lens to regain accommodation. J. Biophoton. 3 (5-6): 265-268

11. Rabbett RB (2007) Bennett \& Rabbetts' clinical visual optics. Elsevier/Butterworth Heinemann, Edinburgh

12. Ripken T, Oberheide U, Fromm M, Schumacher S, Gerten G, Lubatschowski H (2008) fs-Laser induced elasticity changes to improve presbyopic lens accommodation. Graefe Arch Clin Exp Ophthalmol 246 (6): 897-906

13. Schumacher S, Oberheide U, Fromm M, Ripken T, Ertmer W, Gerten G, Wegener A, Lubatschowski H (2009). Femtosecond laser induced flexibility change of human donor lenses. Vision Res 49 (14): 1853-1859

14. Stachs O, Schumacher S, Hovakimyan M, Fromm M, Heisterkamp A, Lubatschowski H, Guthoff R (2009) Visualization of femtosecond laser pulse-induced microincisions inside crystalline lens tissue. J Refract Surg, 35 (11): 1979-1983

15. Strenk SA, Semmlow JL, Strenk LM, Munoz P, Gronlund-Jacob J, DeMarco JK (1999) Age-related changes in human ciliary muscle and lens: A magnetic resonance imaging study. Invest Ophth Vis Sci, 40 (6): 1162-1169

16. Weeber HA, Eckert G, Pechhold W, van der Heijde GL (2007). Stiffness gradient in the crystalline lens. Graefe Arch Clin Exp Ophthalmol, 245 (9): 1357-1366.

17. Weeber HA, van der Heijde GL (2007) On the relationship between lens stiffness and accommodative amplitude. Experimental Eye Research, 85 (5): 602-607

18. Wilde GS, Burd HJ, Judge SJ (2012) Shear modulus data for the human lens determined from a spinning lens test. Experimental Eye Research, 97 (1): 36-48 